\title{
Cholestatic models induced by lithocholic acid and $\alpha$-naphthylisothiocyanate: Different etiological mechanisms for liver injury but shared JNK/STAT3 signaling
}

\author{
GANGMING XU ${ }^{1}$, MANYUN DAI ${ }^{1}$, XIUTING ZHENG ${ }^{1}$, HANTE LIN $^{1}$, AIMING LIU $^{1}$ and JULIN YANG ${ }^{2}$ \\ ${ }^{1}$ Department of Pharmacology, Medical School of Ningbo University, Ningbo, Zhejiang 315211; \\ ${ }^{2}$ Department of Basic Nutrition, Ningbo College of Health Sciences, Ningbo, Zhejiang 315100, P.R. China
}

Received October 24, 2019; Accepted April 9, 2020

DOI: $10.3892 / \mathrm{mmr} .2020 .11210$

\begin{abstract}
ANIT) is used to induce intrahepatic cholestasis and it is frequently used for investigations into the disease mechanism. The lithocholic acid (LCA) cholestatic model has also been extensively used in various studies; however, to the best of our knowledge, a comparative study determining the hepatotoxic mechanisms induced by these two models has not been previously conducted. In the present study, ICR mice were treated with ANIT or LCA to induce cholestatic liver injury. Biochemical analysis was used to determine the serum. Alanine aminotransferase (ALT), aspartate aminotransferase (AST), alkaline phosphatase (ALP) and total bile acid (TBA) levels, and histopathological assessment was used to examine the liver tissue. Metabolomic analysis was used for the serum biomarker identification. Reverse transcription-quantitative PCR analysis and western blotting were used to analyze the inflammation biomarkers. The serum metabolome of the ANIT group clustered away from of the LCA group, which was demonstrated by the different modifications of the BA components. ALP level was found to be preferentially increased in the ANIT group from 24 to $48 \mathrm{~h}$. Total BA levels was only increased in the ANIT group at $24 \mathrm{~h}$. In contrast, AST and ALT activity levels were preferentially increased in the LCA group. The bile ducts in the hepatic tissues of the ANIT group were observed to be severely dilated, whereas the presence of edematous hepatocytes around the necrotic lesions and neutrophil infiltration were identified in the LCA group. The expression levels of cholesterol $7 \alpha$-hydroxylase and sterol $12 \alpha$-hydroxylase genes were significantly downregulated in the ANIT group compared with the LCA group, where a stronger adaptation
\end{abstract}

Correspondence to: Dr Julin Yang, Department of Basic Nutrition, Ningbo College of Health Sciences, 51 Xuefu Road, Ningbo, Zhejiang 315100, P.R. China

E-mail: yangjulin2002@126.com

Key words: $\alpha$-naphthylisothiocyanate, lithocholic acid, cholestatic liver injury, etiological mechanisms, JNK/STAT3 signaling of BA metabolism was supported by major differences in the concentration of the BA components. Despite the aforementioned etiological differences in the cholestasis induced by each treatment, the activation of the JNK/STAT3 signaling pathway was similar between the two cholestatic models. In conclusion, these data suggested that the liver injury induced by ANIT may be cholestatic, while the liver injury caused in the LCA model may be hepatocellular. Moreover, the downstream cholestatic liver injury in both models was indicated to be mediated by the JNK/STAT3 signaling pathway.

\section{Introduction}

Primary biliary cholangitis (PBC) and Primary sclerosing cholangitis (PSC) are typical cholestatic disorders, which are characterized by the retention of bile acid (BA) (1). The prevalence of PBC in northern Europe and Asian countries has been estimated to be 7-402 and 400-500 cases per million, respectively $(2,3)$. In Västra Götaland, which is a regional healthcare unit in Sweden, the reported prevalence of PSC was 162 cases per million subjects in 2005 (4). It has also been reported that $>50 \%$ of patients with PSC may not survive without liver transplantation in the late stages of the disease $(5,6)$. Therefore, cholestasis is one of the most difficult diseases to be handled in hepatology research.

The $\alpha$-naphthylisothiocyanate (ANIT) cholestatic model is the most widely used model for investigating cholestatic liver diseases $(7,8)$; for instance, following the administration of one dose of ANIT into mice, significant hepatic injury was observed (9). The STAT3 and NF- $\mathrm{NB}$ signaling pathways have been discovered to serve an important role in this type of cholestatic liver injury (9). In a previous study, upon the treatment of mice with a high dose of ANIT $(300 \mathrm{mg} / \mathrm{kg})$, rapid (15-24 h) cholestasis developed alongside severe cholangiocyte destruction, which was extended to the periportal hepatocytes (10). In addition, in mouse cholestasis induced by one dose of ANIT, $c$-Jun and $c$-Fos transcription was significantly upregulated and phosphorylated (p)-STAT3 activation was also observed (11). In male Sprague-Dawley rats treated with a single dose of ANIT for $24 \mathrm{~h}$, the AMP-activated protein kinase/Farnesoid X receptor (FXR) signaling pathway served a critical role in the disruption of 
BA homeostasis (12). Therefore, the inflammatory pathways involved in cholestatic liver disease remain as the main research topic for investigation.

Lithocholic acid (LCA)-induced cholestasis is a cholestatic model that has been previously used in multiple studies $(13,14)$. For example, C57BL/6 mice were intraperitoneally injected with $250 \mathrm{mg} / \mathrm{kg}$ LCA twice daily for 4 days and the pathological changes of the liver tissues were observed, including severe hepatic necrosis and diffuse vacuolization (13). In C57BL/6 mice treated with a $1 \%$ LCA diet $(\mathrm{g} / \mathrm{g})$ for 4 days, the upregulation of the levels of the inflammatory factors interleukin (Il) $1 \beta$, Il 6 and $I l 10$ were observed (15). In addition, in male mice treated with $125 \mathrm{mg} / \mathrm{kg}$ LCA (b.i.d) for 7 days, $\mathrm{NF}-\kappa \mathrm{B}$ activation was increased, which was accompanied by an elevation in the expression levels of the inflammatory cytokines tumor necrosis factor (Tnf) $\alpha$ and Il6 (16). Finally, in C57BL/6 mice, an intraperitoneal injection of $125 \mathrm{mg} / \mathrm{kg} \mathrm{LCA}$ twice daily for 4 days promoted an increase in $N F-\kappa \mathrm{B}$ gene expression, as well as in Tnf $\alpha$ and Ill $\beta$ levels (17). However, to the best of our knowledge, the comparison of the toxicity and the mechanisms of action between the LCA and ANIT models has not been previously reported.

In the present study, ICR mice were treated with ANIT or LCA to induce cholestatic liver injury. The etiological differences of cholestasis between the two cholestatic models were revealed using metabolomics and traditional approaches. Notably, it was discovered that the JNK/STAT3 signaling pathway mediated the cholestatic liver injury in both models.

\section{Materials and methods}

Chemicals, reagents and assay kits. ANIT and LCA were purchased from Sigma-Aldrich (Merck KGaA). The BA components, taurocholic acid (TCA), tauro- $\alpha$-muricholic acid (T- $\alpha$ MCA), tauro- $\beta$-muricholic acid (T- $\beta$ MCA), tauro- $\omega$-muricholic acid (T- $\omega \mathrm{MCA})$, taurochenodeoxycholic acid (TCDCA), tauroursodeoxycholic acid (TUDCA) and taurodeoxycholic acid (TDCA), were also purchased from Sigma-Aldrich (Merck KGaA). Assay kits for the liver injury markers alanine aminotransferase (ALT; cat. no. 181017101), aspartate aminotransferase (AST; cat. no. 181012101), alkaline phosphatase (ALP; cat. no. 1060-717) and total BA (TBA; cat.no. 1025-717) were purchased from Ruiyuan Biotechnology (http://www.reebio.com/about.html). TRIzol ${ }^{\circledR}$ reagent and cDNA Synthesis kit were both purchased from Thermo Fisher Scientific, Inc. LightCycle 480 SYBR Green I Master mix was purchased from Roche Diagnostics (Shanghai) Co., Ltd. The antibodies against total (t)-JNK (1:1,000; cat. no. 9252) and p-JNK (1:1,000; cat. no. 9912) were purchased from Cell Signaling Technology, Inc. Finally, the primary antibodies against p-p65 (1:5,000; cat. no. ab86299), t-p65 (1:5,000; cat. no. ab32536), t-STAT3 (1:5,000; cat. no. ab109085), p-STAT3 (1:5,000; cat. no. ab76315) and GAPDH (1:5,000; cat. no. ab181602) were obtained from Abcam. Secondary antibodies [IgG H\&L (horseradish peroxidase)] used were obtained from Abcam (1:2,000; cat. no. ab205718).

Animals and treatment. The animal studies were performed following the approval of the protocol by the Institutional Animal Care and Use Committee (approval no. IACUC
201707-138) at Ningbo University (Zhejiang, China). In total, 15 male ICR mice (age, 5-7 weeks; weight, $20 \pm 5 \mathrm{~g}$ ) were purchased from Slac Laboratory Animal (Shanghai, China) Co., Ltd. Prior to the experiments, the mice were housed at the Medical School of Ningbo University Animal Services Unit at $23 \pm 1^{\circ} \mathrm{C}$, with a relative humidity of $60-70 \%$, a light/ dark cycle of $12 \mathrm{~h}$ and with free access to water and standard mouse chow. The mice were kept in standard cages $(n=5)$ with aspen bedding. The ICR mice were assigned to the following three groups: Control group, LCA group and ANIT group. The LCA group was orally treated with $150 \mathrm{mg} / \mathrm{kg}$ LCA in corn oil two times a day. The animals were sacrificed $12 \mathrm{~h}$ following the 5th treatment. The ANIT group was treated with $75 \mathrm{mg} / \mathrm{kg}$ ANIT in corn oil once by oral gavage and the animals were sacrificed after $48 \mathrm{~h}$. The blood samples $(50 \mu \mathrm{l})$ were collected in the two models by tail bleeding at 0,12 , 24 and $36 \mathrm{~h}$ following the 1 st dose to monitor the progress of toxicity.

When the treatment finished, euthanasia was performed using $\mathrm{CO}_{2}$ (20\% volume displacement rate/min) at a flow rate of $2 \mathrm{l} / \mathrm{min}$. Ventilation was maintained for 1-2 min until no breathing was observed in the mice, and corneal reflexes and body stiffness were also checked to confirm mortality. Cervical dislocation was used as the secondary means of euthanasia for those mice that survived. Serum and liver samples were collected in a same manner as described previously (18). A section of freshly isolated liver tissues (1/5 of the liver tissues) was excised and immediately fixed in $10 \%$ neutral buffered formalin at room temperature for at least $24 \mathrm{~h}$ after a brief wash with PBS. The remaining liver tissues for reverse transcription-quantitative PCR (RT-qPCR; 2/5 of the liver tissues) and western blotting (2/5 of the liver tissues) were flash-frozen in liquid nitrogen and stored at $-80^{\circ} \mathrm{C}$.

Biochemical analysis. The blood samples were centrifuged at $800 \times \mathrm{g}$ at $4^{\circ} \mathrm{C}$ for $10 \mathrm{~min}$ to obtain the serum. The serum ALT, AST, ALP and TBA levels were analyzed using the kits at $0,24,36$ and $48 \mathrm{~h}$, according to the manufacturer's protocol, and a Multiskan GO microplate reader (Thermo Fisher Scientific, Inc.).

Histopathological assessment. The liver fixed tissues were dehydrated in an ascending series of alcohol (70, 80, 90 and $100 \%$ ) and washed with xylene. The tissues were embedded in paraffin at $60^{\circ} \mathrm{C}$ and cooled to $-20^{\circ} \mathrm{C}$, prior to being cut into 4- $\mu \mathrm{m}$ sections. The sections were stained with hematoxylin for $3 \mathrm{~min}$ and eosin for $2 \mathrm{~min}$ at room temperature. The stained liver sections were visualized under an Olympus BX51 light microscope (Olympus Corporation) at x40 and x400 magnification and ten serial sections per preparation were analyzed blindly by a pathologist.

Serum metabolome analysis. The analysis of the serum metabolome was performed as described previously (18). After being processed by Marker Lynx 4.1 (Waters Corporation), a data matrix of peak areas organized by retention time and $\mathrm{m} / \mathrm{z}$ was generated and then exported into SIMCA-P 13.0.3 (Umetrics; Sartorius AG; https://umetrics.com/products/simca) for pareto transformation. Then, unsupervised principal component analysis was used to produce score plot. Orthogonal projection 

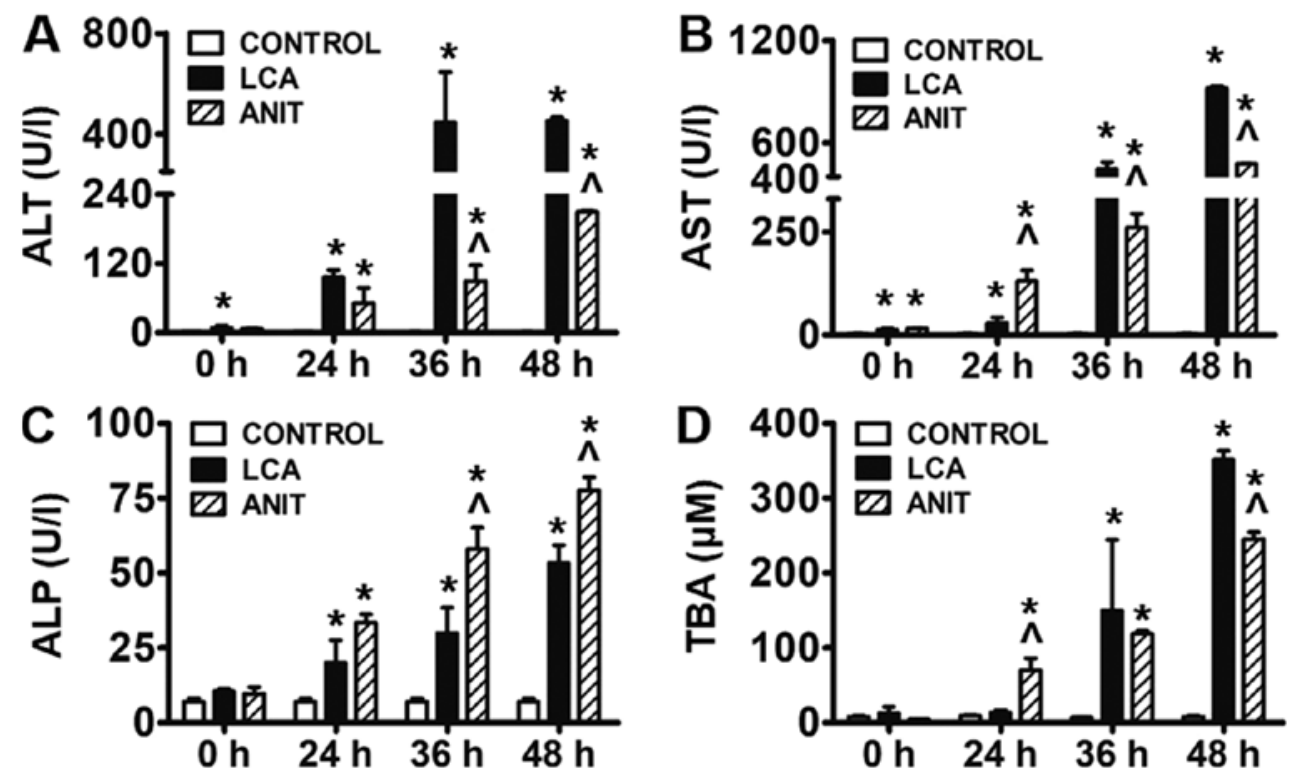

Figure 1. Biochemical markers indicating the hepatotoxicity following ANIT and LCA treatment. (A) ALT level in the control, LCA and ANIT groups. (B) AST level in the control, LCA and ANIT groups. (C) ALP level in the control, LCA and ANIT groups. (D) TBA level in the control, LCA and ANIT groups. The data were expressed as the mean \pm SD. ${ }^{*} \mathrm{P}<0.05$ vs. control group; ${ }^{\wedge} \mathrm{P}<0.05$ vs. LCA group. ANIT, $\alpha$-naphthylisothiocyanate; LCA, lithocholic acid; ALT, alanine aminotransferase; AST, aspartate aminotransferase; ALP, alkaline phosphatase; TBA, total bile acid.

to latent structures discriminant analysis was exploited to produce the loading S-plot of LCA-treated and ANIT-treated mice vs. the control mice where the BAs contributing to the pattern recognition were exhibited. Accurate molecular weights of known BA components were used to match the contributing items, which were determined to be TCA, T- $\alpha / \beta / \omega M C A$, TCDCA, TUDCA and TDCA by subsequent comparison of the MS/MS spectra with those of the authentic compounds. The relative abundance of these BAs in the serum were normalized by comparing their peak areas with those of internal standard. Their abundance in serum was expressed as fold changes of LCA-treated and ANIT-treated mice vs. the control groups. In the score (S)-plot, the five components were identified as TCA, T- $\alpha / \beta / \omega \mathrm{MCA}$, TCDCA, TUDCA and TDCA by comparison with authentic standards. T- $\alpha \mathrm{MCA}$, T- $\beta$ MCA and T- $\omega$ MCA could not be separated at baseline during chromatographic analysis and were consequently combined and named as T- $\alpha / \beta / \omega \mathrm{MCA}$.

$R T$ - $q P C R$. The liver tissues (20 mg) were homogenized using TRIzol ${ }^{\circledR}$ reagent following the manufacturer's protocol. Then pure chloroform was added for $5 \mathrm{~min}$ to extract total RNA at room temperature. Centrifugation was performed at 3,200 x g for $20 \mathrm{~min}$ at $4^{\circ} \mathrm{C}$ and precipitated with $75 \%$ ethanol. Total RNA was quantified using the Multiskan GO microplate reader. Total RNA was reverse transcribed into cDNA using a RT system (20 $\mu \mathrm{l}$ ) including the following: $4 \mu \mathrm{l}$ 5X reaction buffer, $1 \mu \mathrm{g}$ total RNA, $1 \mu \mathrm{l}$ Oligo dT18, $1 \mu \mathrm{l}$ random primer, $2 \mu \mathrm{l}$ dNTPs (10 $\mathrm{mM})$ mix, $1 \mu 1$ reverse transcriptase, $1 \mu 1$ RNase inhibitor and $2 \mu 1$ RNase-free water. The RT temperature protocol was as follows: Annealing at $25^{\circ} \mathrm{C}$ for $5 \mathrm{~min}$, extension at $42^{\circ} \mathrm{C}$ for $1 \mathrm{~h}$, inactivation at $70^{\circ} \mathrm{C}$ for $5 \mathrm{~min}$ and chilling at $4^{\circ} \mathrm{C}$ for holding. qPCR was subsequently performed using a $5 \mu \mathrm{l}$ qPCR system in a 384-well plate, which included $1 \mu$ l total cDNA, 2.5 $\mu$ l SYBR-Green I Master Mix, $0.2 \mu$ l forward and reverse primer, and $2 \mu 1 \mathrm{RNase}$-free water. The following primer pairs used for the qPCR are listed in Table SI. The following thermocycling conditions were used for the qPCR: $95^{\circ} \mathrm{C}$ for $10 \mathrm{sec}$, $55^{\circ} \mathrm{C}$ for $10 \mathrm{sec}$ and $72^{\circ} \mathrm{C}$ for $15 \mathrm{sec}$. Expression levels were quantified using the $2^{-\Delta \Delta \mathrm{Cq}}$ method (19) and normalized to $18 \mathrm{~S}$ ribosomal RNA. The expression levels in the control group were set to 1, and the data of the LCA group and ANIT group were normalized and expressed as relative expression.

Western blotting. Livers of three mice in each group were used in protein analysis. Total protein was extracted from freshly cut liver tissues using RIPA lysis buffer (Beijing Solarbio Science \& Technology Co., Ltd.; 1:10; g/v), supplemented with 1\% phenylmethylsulfonyl fluoride, using a MagNA Lyser instrument (Roche Applied Science). Tissue debris was removed by centrifugation at $10,000 \mathrm{x}$ g at $4^{\circ} \mathrm{C}$ for $5 \mathrm{~min}$. Total protein was quantified using a bicinchoninic acid assay kit (Beyotime Institute of Biotechnology) and adjusted to $5 \mathrm{mg} / \mathrm{ml}$. 2X loading buffer (Beijing Solarbio Science \& Technology Co., Ltd.) was added to the samples (volume-volume, 1:1), which were boiled for $5 \mathrm{~min}$ at $100^{\circ} \mathrm{C}$. Then, $25 \mu \mathrm{g}$ protein was separated via SDS-PAGE (10\% separating gel; 5\% spacer gel). The separated proteins were transferred onto PVDF membranes and blocked for $4 \mathrm{~h}$ with $5 \%$ fat-free milk in TBS- $0.1 \%$ Tween-20 at room temperature. The membranes were incubated overnight at $4^{\circ} \mathrm{C}$ with the following primary antibodies: Anti-t-JNK $(1: 1,000)$, anti-p-JNK $(1: 1,000)$, anti-p-p65 (1:5,000), anti-t-p65 (1:5,000), anti-t-STAT3 (1:5,000), anti-p-STAT3 $(1: 5,000)$ and anti-GAPDH $(1: 5,000)$. Following the primary antibody incubation, the membranes were incubated with secondary antibodies $(1: 2,000)$ for $1.5 \mathrm{~h}$ at room temperature. Protein bands were visualized by addition of ECL reagents (Advansta, Inc.) and recorded using a Tanon 4200SF chemiluminescent imaging system (Beijing Solarbio Science \& Technology Co., Ltd.). Protein expression levels were semi-quantified using ImageJ 1.8.0 software (National Institutes of Health). 
Statistical analysis. Statistical analysis was performed using SPSS version 23 software (IBM Corp.) and all data are expressed as the mean \pm SD. SIMCA-P 13.0.3 (Umetrics; Sartorius AG) was used for multivariate data analysis (20). Experiments were repeated three times. Statistical differences were determined using a one-way ANOVA followed by Tukey's post hoc test for multiple comparisons. $\mathrm{P}<0.05$ was considered to indicate a statistically significant difference.

\section{Results}

Levels of the liver injury biomarkers differ between the two cholestatic models. ALT and AST levels are frequently used as an indication of liver injury (21). The results revealed that ALT and AST levels were significantly increased in both the ANIT and LCA groups compared with the control group in a time-dependent manner (Fig. 1A and B). Furthermore, ALT levels were significantly increased in the LCA group compared with those in the ANIT group at 36 and $48 \mathrm{~h}$ (Fig. 1A). AST levels were significantly lower in the LCA group compared with the ANIT group at $24 \mathrm{~h}$ (Fig. 1B). However, the levels of AST were significantly higher in the LCA group compared with the ANIT group at 36 and $48 \mathrm{~h}$ (Fig. 1B).

The levels of ALP and TBA were significantly increased in the ANIT and LCA groups compared with the control group from 24-48 h (Fig. 1C and D). Moreover, ALP levels in the ANIT group were significantly higher compared with the LCA group at 36 and $48 \mathrm{~h}$ (Fig. 1C). The levels of TBA were also significantly increased in the LCA group compared with those in the ANIT group at $48 \mathrm{~h}$ (Fig. 1D).

The histopathological data revealed that the control group exhibited a normal microstructure and histology (Fig. 2A and B). The LCA group exhibited more necrotic areas compared with the ANIT group (Fig. $2 \mathrm{C}$ and D). The LCA group was demonstrated to have edematous hepatocytes around the necrotic lesions, with the presence of several vacuoles, infiltrating neutrophils and a mild dilation of the bile ducts (Fig. 2E, G and I). However, the ANIT group exhibited signs of severely dilated bile ducts (Fig. 2F), mild edema, necrotic lesions, the infiltration of neutrophils (Fig. 2H) and severe hepatic sinus congestion (Fig. 2J).

Serum metabolome is affected by the BA components. In the principal component analysis, samples from mice that were treated with LCA and ANIT were classified into independent groups from those in the control group. Their movement direction indicated the different modifications of the serum metabolome in the two models (Fig. 3A).

In the S-plot, a comparison of the contributing components in the two models was performed and the five following components were identified in comparison with commercial pure substances: TCA, T- $\alpha / \beta / \omega \mathrm{MCA}$, TCDCA, TUDCA and TDCA (Fig. 3B). The diagrams associated with the identification of TCA are presented in Fig. 4 and those associated with the identification of the other components (T- $\alpha / \beta / \omega \mathrm{MCA}$, TCDCA, TUDCA and TDCA) are presented in Figs. S1-4. The levels of these components (TCA, T- $\alpha / \beta / \omega \mathrm{MCA}$, TCDCA, TUDCA and TDCA) were significantly increased by $41-$, 70-, 938-, 202- and 490-fold, respectively, in the LCA group compared with the control group (Fig. 5). However, their
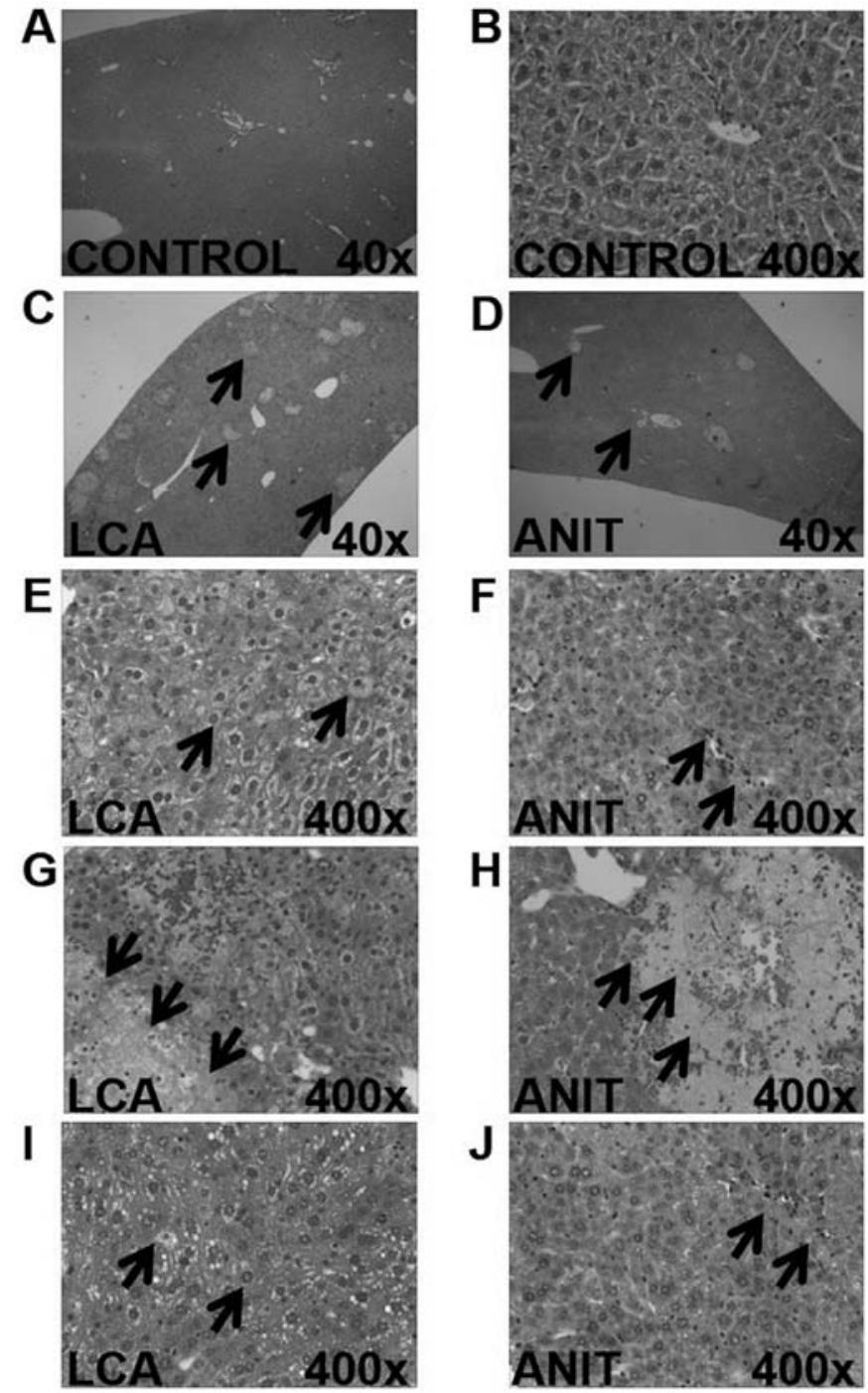

Figure 2. Histopathological analysis of liver tissues to determine the liver injury in the ANIT and LCA group. H\&E staining at magnification of (A) $\times 40$ and (B) $\times 400$ in the control group. H\&E staining at magnification of $\mathrm{x} 40$ in (C) LCA group and (D) ANIT group. (E) H\&E staining of liver tissues in the LCA group at magnification of $x 400$. (F) H\&E staining of liver tissues in the ANIT group at magnification of $x 400$. (G) H\&E staining of liver tissues in the LCA group at magnification of $x 400$. (H) $\mathrm{H} \& \mathrm{E}$ staining of liver tissues in the ANIT group at magnification of $x 400$. (I) $H \& E$ staining of liver tissues in the LCA group at magnification of $x 400$. (J) H\&E staining of liver tissues in the ANIT group at magnification of $x 400$. Liver tissue histological damage is indicated by black arrows. ANIT, $\alpha$-naphthylisothiocyanate; LCA, lithocholic acid; H\&E, hematoxylin and eosin.

increases were estimated to be only 30-, 11-, 32, 4- and 7-fold, respectively, in the ANIT group (Fig. 5). All the other components, except TCA, demonstrated significant differences between the two groups.

BA metabolism is altered in the two cholestatic models. The expression levels of the BA synthesis genes were decreased in the two cholestatic groups. The transcription of cholesterol $7 \alpha$-hydroxylase (Cyp $7 a l)$ and sterol $12 \alpha$-hydroxylase (Cyp $8 b 1$ ) was significantly decreased in the ANIT group compared with the control and LCA groups; Cyp7al and Cyp $8 b 1$ expression levels were decreased by 98 and $94 \%$, respectively, compared with the control group (Fig. 6A and B). In the LCA group, Cyp7al expression levels were significantly decreased by $65 \%$ 

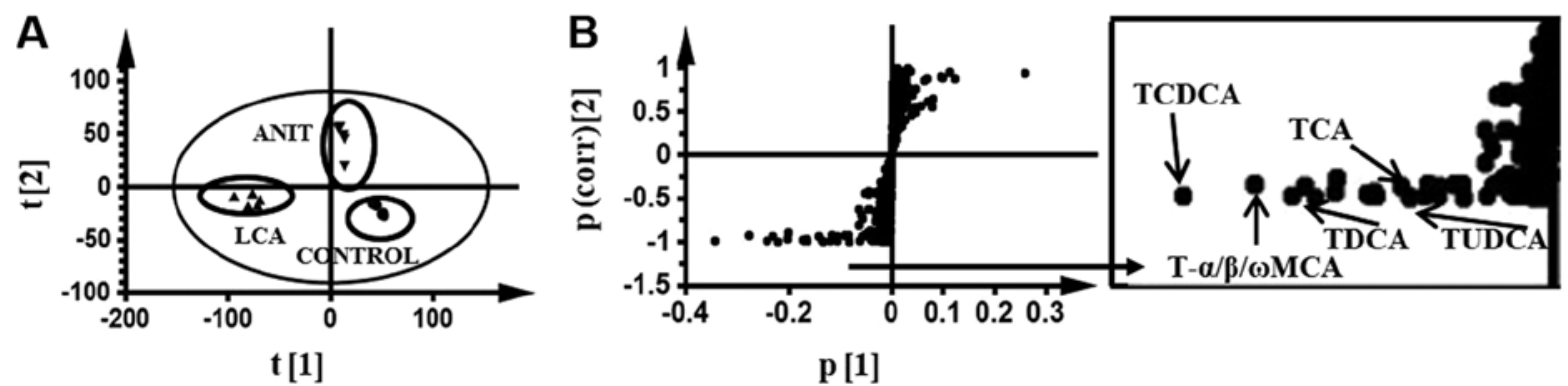

Figure 3. Multivariate data analysis of the serum metabolome in mice dosed ANIT and LCA. (A) S-plot of serum metabolites in ANIT and LCA treated mice vs. the control group by principle component analysis. (B) S-plot of OPLS-DA recognized serum metabolome in ANIT-treated mice vs. LCA-treated mice, in which the contribution of the identified BA components was indicated. Each point in the panel A represented an individual mouse serum sample and the points in panel $\mathrm{B}$ represented contributing metabolites. The $\mathrm{t}[1]$ and $\mathrm{t}[2]$ represent principal components 1 and 2, respectively. The $\mathrm{p}(\mathrm{corr})[1]$ represents the interclass difference and $\mathrm{p}[1]$ represents the relative abundance of the ions. ANIT, $\alpha$-naphthylisothiocyanate; LCA, lithocholic acid; S-plot, score plot; OPLS-DA, orthogonal projection to latent structures discriminant analysis; BA, bile acid; T- $\alpha / \beta / \omega \mathrm{MCA}$, tauro- $\alpha / \beta / \omega$-muricholic acid; TCDCA, taurochenodeoxycholic acid; TUDCA, tauroursodeoxycholic acid; TDCA, taurodeoxycholic acid; TCA, taurocholic acid.
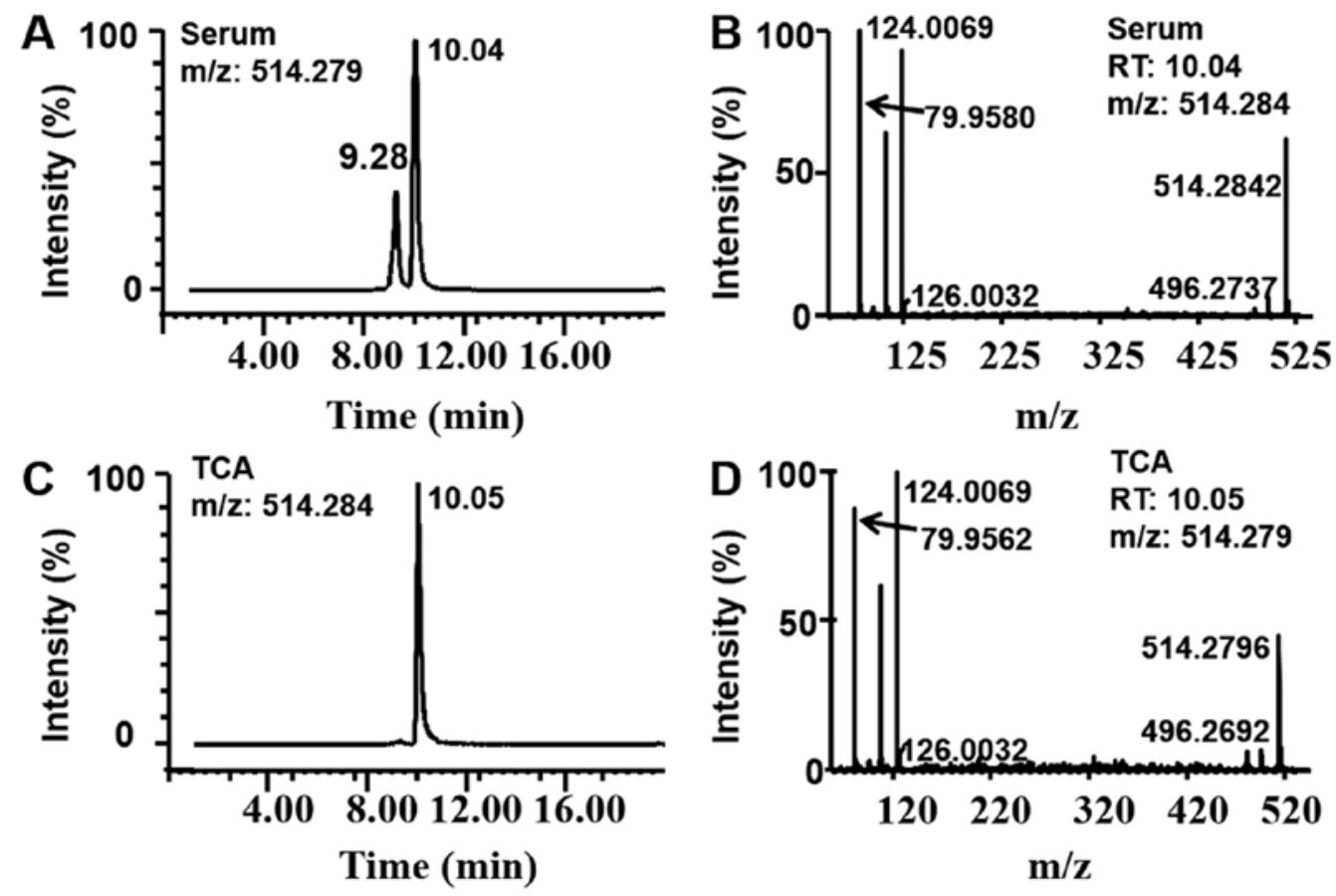

Figure 4. Targeted metabolomic analysis of the serum and biomarker identification in mice treated with ANIT or LCA. (A) TIC chromatogram of m/z 514.279 in the mouse serum sample. (B) MS/MS spectra of the peak with a RT of 10.04 in the mouse serum in part A. (C) TIC chromatogram of authentic TCA when the fragmentation was performed at m/z 514.284. (D) MS/MS spectra of the peak with a RT of 10.05 in part C. ANIT, a-naphthylisothiocyanate; LCA, lithocholic acid; TIC, total ion count; TCA, taurocholic acid; RT, retention time.

compared with the control group; however, Cyp $8 b 1$ expression levels were not significantly altered (Fig. 6A and B). This effect was possibly associated with the different adaptations caused by the BA component. In addition, organic anion transporting polypeptide (Oatp) 1 mRNA expression levels were significantly decreased by $54 \%$ (Fig. 6C), whereas Oatp 2 mRNA expression levels were not significantly altered in the ANIT group compared with the control group (Fig. 6D). Notably, in the LCA group, Oatp1 mRNA expression levels were significantly decreased by $71 \%$ (Fig. 6C) and Oatp 2 mRNA expression levels were significantly decreased by $81 \%$ compared with the control group (Fig. 6D).

The expression levels of the BA transporter genes were also increased in the two groups. Briefly, multidrug resistance protein $(M d r) 2$ mRNA expression levels were significantly increased by 10 -fold in the ANIT group compared with the control group (Fig. 6E). Moreover, a 158-fold increase was noted in the LCA group compared with the control group (Fig. 6E). In addition, Mdrla mRNA expression levels were significantly increased by 15 -fold in the ANIT group, while they were significantly increased by 1.6 -fold in the LCA group compared with the control group (Fig. 6F). Finally, multidrug resistance-related protein ( $M r p) 4$ mRNA expression level and organic solute transporter- $\beta$ (Ostb) mRNA expression levels were both significantly increased in the LCA and ANIT groups compared with the control group; however, no significant difference was identified in the mRNA expression levels between the two cholestatic groups (Fig. 6G and H). 


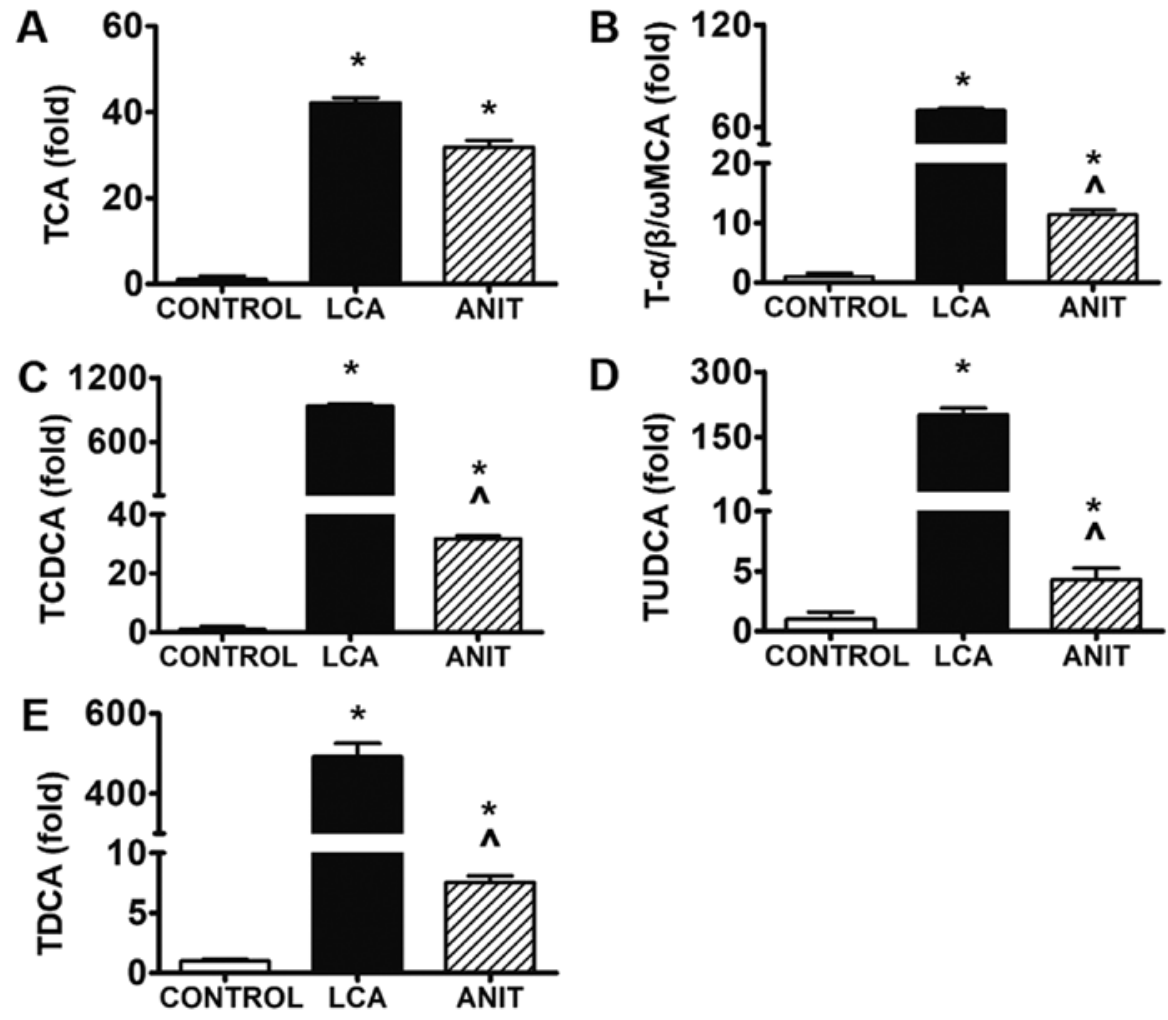

Figure 5. Relative abundance of BA components identified in ANIT and LCA mice. The relative abundance of (A) The relative abundance of TCA in the control, LCA and ANIT groups. (B) The relative abundance of T- $\alpha / \beta / \omega \mathrm{MCA}$ in the control, LCA and ANIT groups. (C) The relative abundance of TCDCA in the control, LCA and ANIT groups. (D) The relative abundance of TUDCA in the control, LCA and ANIT groups. (E) The relative abundance of TDCA in the control, LCA and ANIT groups. The data were expressed as the mean \pm SD. ${ }^{~} \mathrm{P}<0.05$ vs. control group; ${ }^{\wedge} \mathrm{P}<0.05$ vs. LCA group. BA, bile acid; ANIT, $\alpha$-naphthylisothiocyanate; LCA, lithocholic acid; TCA, taurocholic acid; T- $\alpha / \beta / \omega \mathrm{MCA}$, tauro- $\alpha / \beta / \omega$-muricholic acid; TCDCA, taurochenodeoxycholic acid; TUDCA, tauroursodeoxycholic acid; TDCA, taurodeoxycholic acid.

Induction of inflammation in the two models is mediated by the JNK/STAT3 signaling pathway. The mRNA expression levels of the proinflammatory factors $I l 10, c$-Fos, Il6, suppressor of cytokine signaling 3 (Socs3), fibrinogen $\alpha$ chain (Fga) and fibrinogen $\beta$ chain (Fgb) were significantly increased in the LCA and ANIT groups compared with the control group (Fig. 7A-F), suggesting a downstream inflammatory response in the LCA and ANIT groups. Moreover, the mRNA expression levels of IllO, c-Fos, Il6, Socs3, Fga and $\mathrm{Fg} b$ were significantly increased in the LCA group compared with the ANIT group (Fig. 7A-F). Although, $c$-Jun mRNA levels were significantly increased in the two cholestatic groups compared with the control, no significant difference was observed between these two groups (Fig. 7G). However, the mRNA expression levels of Tnf $\alpha$ were not significantly different between the two cholestatic groups and the control group (Fig. 7H).

In western blotting, there was slight difference between the ratio of p-p65/t-p65 in control, LCA and ANIT groups (3.2, 1.5 and 2.5; Fig. 8). These patterns were consistent with the differences identified in the expression levels of $T n f \alpha$ mRNA. Therefore, it was speculated that the NF- $\kappa B$ pathway may not serve a critical role in cholestatic inflammation. However, there was significant difference in the ratio of $\mathrm{p}-\mathrm{JNK} / \mathrm{t}-\mathrm{JNK}$ in control, LCA and ANIT groups (0.1, 1.0 and 0.7$)$, which was significantly increased in the LCA and ANIT groups compared with the control group. In addition, there was a significant difference in the ratio of p-STAT3/t-STAT3 in control, LCA and ANIT groups $(0.2,0.9$ and 0.3$)$, which was significantly increased in the LCA and ANIT groups compared with the control group (Fig. 8). Considering that STAT3 is the transcription factor downstream of JNK (22), the activation of the JNK/STAT3 signaling axis was suggested to be a main contributing factor for the liver injury of the two models.

\section{Discussion}

During ANIT-induced cholestasis, a higher biliary concentration level of ANIT was discovered to be toxic to cholangiocytes (23). Subsequently, the blocked excretion of BA was discovered to increase the BA load in the hepatocytes and induce hepatotoxicity (24). The accumulation of LCA has been implicated as a major factor contributing to cholestasis due to its inherent cytotoxicity (25). In the present study, the ANIT group was treated with a single dose of ANIT $(75 \mathrm{mg} / \mathrm{kg})$, which was at the same level as typically used in mice $(9,11)$, whereas the LCA group included mice that were orally administered five times with LCA $(150 \mathrm{mg} / \mathrm{kg}$; b.i.d), which was considered a low level dose compared with the published dose range $(13,15,16)$. This dose level was validated in this study and could stably produce cholestatic liver injury without lethal action. Moreover, the levels of the liver injury biomarkers ALT and AST were higher in the LCA group, while the levels of the cholestatic biomarker ALP were higher in the ANIT group. The pathological analysis revealed that the LCA group exhibited edematous hepatocytes 

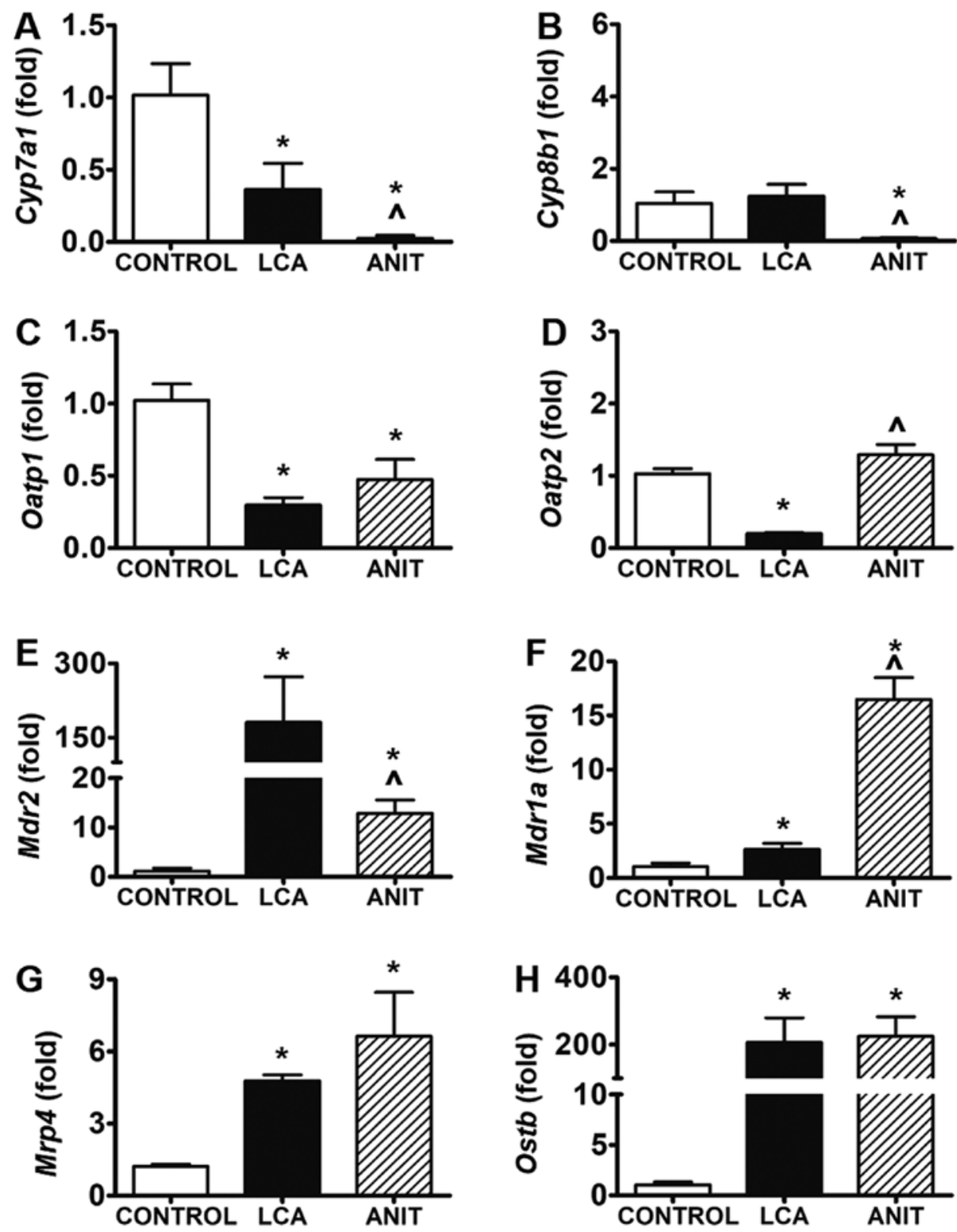

Figure 6. Expression levels of genes involved in bile acid synthesis, uptake and export. (A) Cyp7a1 mRNA expression in the control, LCA and ANIT groups. (B) Cyp8b1 mRNA expression in the control, LCA and ANIT groups. (C) Oatp1 mRNA expression in the control, LCA and ANIT groups. (D) Oatp2 mRNA expression in the control, LCA and ANIT groups. (E) Mdr2 mRNA expression in the control, LCA and ANIT groups. (F) Mdrla mRNA expression in the control, LCA and ANIT groups. (G) Mrp4 mRNA expression in the control, LCA and ANIT groups. (H) Ostb mRNA expression in the control, LCA and ANIT groups. The mRNA expression levels were analyzed using reverse transcription-quantitative PCR and normalized to 18S ribosomal RNA. Data from the liver samples was collected following ANIT and LCA treatment. mRNA expression levels in the vehicle-treated control mice were set as 1 and the results were expressed as the mean $\pm \mathrm{SD}$. N=5. $\mathrm{P}<0.05$ vs. control group; ${ }^{\wedge} \mathrm{P}<0.05$ vs. LCA group. ANIT, $\alpha$-naphthylisothiocyanate; LCA, lithocholic acid; Cyp7a1, cholesterol $7 \alpha$-hydroxylase; Cyp8b1, sterol 12 $\alpha$-hydroxylase; Oatp1, organic anion transporting polypeptide 1; Oatp2, organic anion transporting polypeptide 2; Mdr2, multidrug resistance protein 2; Mdr1a, multidrug resistance protein 1a; Mrp4, multidrug resistance-related protein 4; Ostb, organic solute transporter- $\beta$.

around the necrotic lesions with the presence of several vacuoles in the hepatic tissues. In contrast, the ANIT group exhibited severely dilated bile ducts and severe hepatic sinus congestion. The toxicity features were consistent with the perspective that ANIT acted on the biliary epithelial cells, whilst the injury induced by LCA was considered to be hepatocellular $(26,27)$.

LCA is absorbed in the colon, transported to the liver and subsequently metabolized in the hepatocytes (25). The metabolites of LCA include the major metabolite murideoxycholic acid, isolithocholic acid and 3-keto-5-cholanic acid, whereas 6-ketolithocholic acid and ursodeoxycholic acid are considered minor metabolites (25). The enzymes involved in phase I metabolism include cytochrome P450 (CYP)2C, CYP3A, CYP2A and certain non-cytochrome P450 enzymes (25). In the present study, the measured BA components were all higher in the LCA group compared with the ANIT group. However, these components were not the aforementioned metabolites of LCA. In addition, the metabolites of LCA have been reported to be protective for liver function $(28,29)$. Therefore, it was hypothesized that the metabolites of LCA may be the main contributors to the toxicity observed, although insufficient evidence is presented. However, it is also possible that the metabolites of LCA compete against the metabolism and transport of 

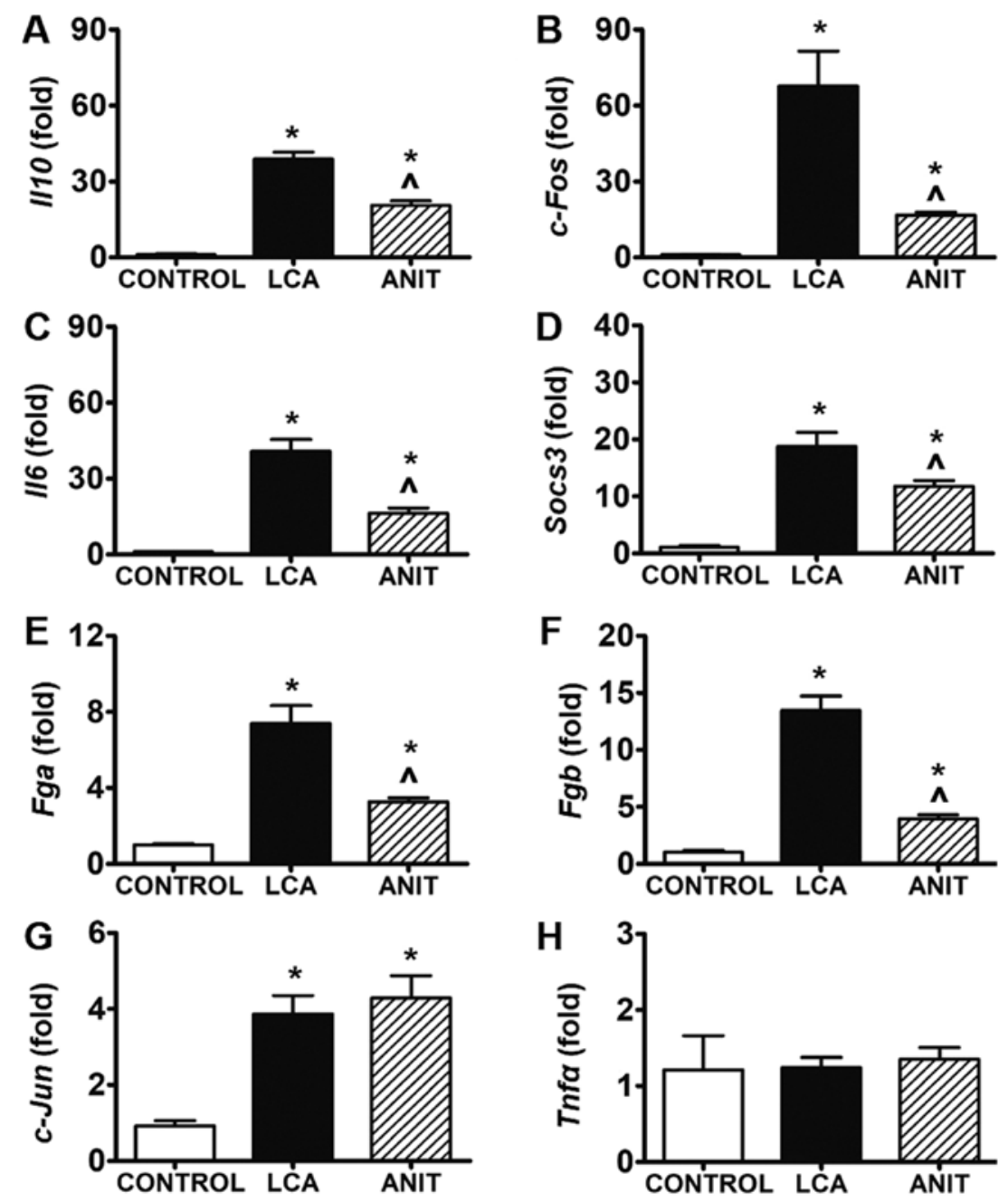

Figure 7. Differentially regulated genes involved in inflammation. mRNA expression levels of (A) Il10 mRNA expression in the control, LCA and ANIT groups . (B) c-Fos mRNA expression in the control, LCA and ANIT groups. (C) Il6 mRNA expression in the control, LCA and ANIT groups. (D) Socs3 mRNA expression in the control, LCA and ANIT groups. (E) Fga mRNA expression in the control, LCA and ANIT groups. (F) Fgb mRNA expression in the control, LCA and ANIT groups. (G) c-Jun mRNA expression in the control, LCA and ANIT groups. (H) Tnfo mRNA expression in the control, LCA and ANIT groups. The mRNA levels were analyzed using reverse transcription-quantitative PCR and normalized to $18 \mathrm{~S}$ ribosomal RNA. The mRNA expression levels in the vehicle-treated control mice were set as 1 and the results were expressed as the mean $\pm \mathrm{SD}$. $\mathrm{N}=5$. ${ }^{*} \mathrm{P}<0.05$ vs. control group; ${ }^{\wedge} \mathrm{P}<0.05$ vs. LCA group. ANIT, $\alpha$-naphthylisothiocyanate; LCA, lithocholic acid; Il10, interleukin 10; I16, interleukin 6; Socs3, suppressor of cytokine signaling 3; Fga, fibrinogen $\alpha$ chain; Fgb, fibrinogen $\beta$ chain; Tnf $\alpha$, tumor necrosis factor $\alpha$.

the other BA components, which would subsequently lead to cholestasis and liver injury (25). Based on this evidence, it was hypothesized that the induced liver injury in the LCA group was associated with the accumulation of toxic BA due to LCA administration, rather than due to the direct action of the LCA metabolites.

With regards to the metabolism of BAs, it has been previously reported that the expression levels of Cyp $7 \alpha 1$ and $C y p 8 b 1$ were reduced following the administration of the ANIT model, which was mediated by FXR (30). In the present study, TBA levels were significantly increased in the ANIT group within the first $24 \mathrm{~h}$. In contrast to these findings, TBA levels were significantly increased between $36-48 \mathrm{~h}$ in the LCA group. Therefore, a more potent adaptation of BA metabolism was identified in the ANIT group compared with that in the LCA group. As expected, the expression levels of Cyp7al and Cyp $8 b 1$ mRNA in the ANIT group were lower compared with the LCA group. The expression levels of inflammatory factors (IllO, c-Fos, Il6, Socs3, Fga and Fgb) were also increased to a higher extent in the LCA group, further suggesting the weaker adaptation to BA metabolism. TUDCA is an antagonist of the nuclear factor FXR, whereas TCA is an agonist of the same receptor, which mediates the adaptation to BA metabolism (31). In the present study, the modification of TUDCA (202-fold vs. 3-fold) was considerably higher compared with the TCA (41-fold vs. 30 -fold) in the LCA group compared with the ANIT group. The biochemical responses, pathological responses, toxic BA levels and the adaptation mode exhibited a significant association with each other following LCA and ANIT treatment.

Several known inflammatory pathways are associated with cholestatic liver injury. In a previous study, endoplasmic reticulum stress-related JNK activation was discovered to be associated with LCA-induced hepatocyte apoptosis (32). In an ANIT-induced cholestatic model, the JNK pathway was also associated with cholestatic liver injury, whereby the inhibition of JNK prevented the cholestasis-induced liver injury but not cholestasis itself, suggesting the critical role of the JNK 

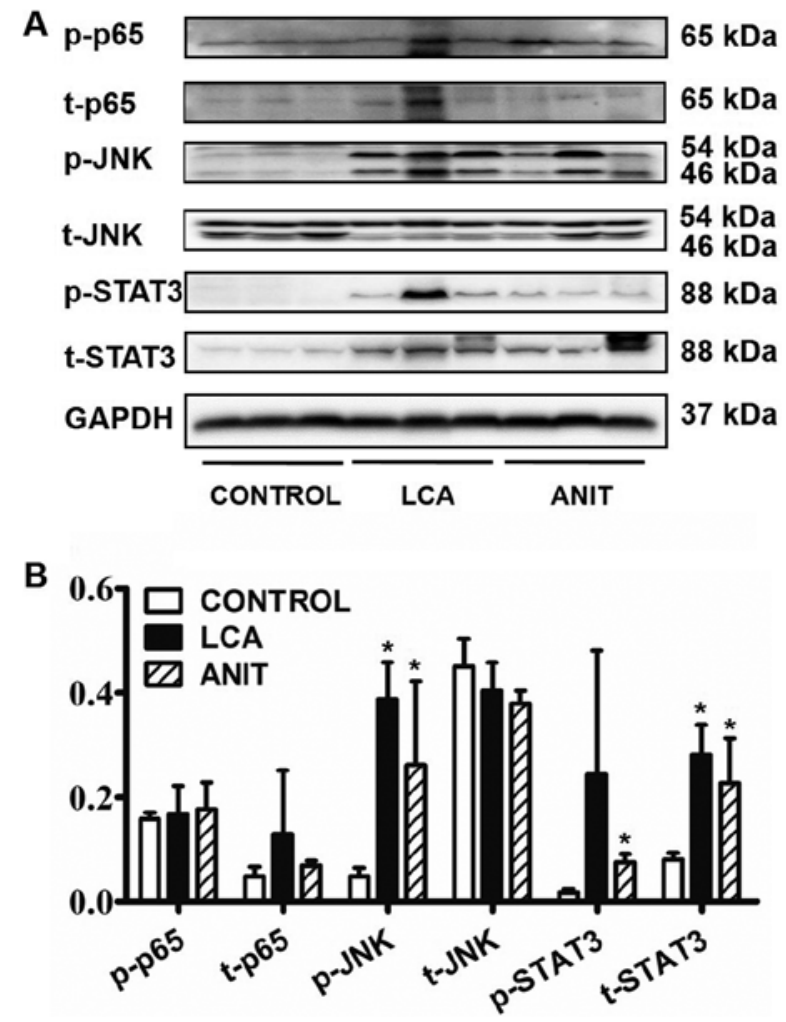

Figure 8. Expression and activation of transcription factors JNK, NF- $\mathrm{B}$ and STAT3 associated with genes detected. (A) Western blots of total and phosphorylated JNK, NF- $\mathrm{KB}$ and STAT3 in liver extracts. (B) Semi-quantification of the western blots in panel A for the control, LCA and ANIT groups. Livers of three mice in each group were randomly selected for western blotting Data are presented as the mean $\pm \mathrm{SD}$. ${ }^{*} \mathrm{P}<0.05$ vs. control group. GAPDH was used as a loading control. ANIT, $\alpha$-naphthylisothiocyanate; LCA, lithocholic acid; t-, total; p-, phosphorylated.

signaling pathway in this condition (11). In ANIT-treated mice, cholestatic liver injury was inhibited by chlorogenic acid, which was involved in the inhibition of NF- $\kappa$ B and STAT3 phosphorylation (9). In addition, the treatment of mice with LCA $(125 \mathrm{mg} / \mathrm{kg})$ twice daily for 7 days resulted in the activation of the NF- $\kappa$ B signaling pathway (16). In the present study, the five doses of LCA were administered with an interval of $12 \mathrm{~h}$, which was associated with increasing toxicity over time. The ANIT group was treated with a single dose of ANIT as determined by a previous study (9). In addition, the NF- $\kappa \mathrm{B}$ protein components exhibited a minor activation in the two cholestatic groups, which was associated with the expression of the proinflammatory factor Tnf $\alpha$. In contrast, the expression level of the p-JNK protein was significantly increased in both the LCA and ANIT groups and the expression of the p-STAT3 protein was significantly increased in the ANIT group; their activation was similar between the two groups and consistent with the expression of their target genes (JNK: c-Fos and c-Jun; STAT3: Socs3, Fga and Fgb). The differences identified in the expression levels of the cytokines represented the intensity of toxicity of the models and not the toxicity mechanism behind the two models. Based on the aforementioned evidence, the extent of inflammation in the two models was hypothesized to be mediated by the JNK/STAT3 signaling pathway.

Neutrophils serve a significant role in ANIT-induced liver injury and neutrophil infiltration is considered the critical step in inducing hepatocyte necrosis in cholestatic liver injury (33). The ANIT concentration gradient between the bile and plasma was reported to recruit the neutrophils to the periportal regions and release lysosomal proteases to damage the liver cells (33). In addition, in a LCA-induced model, intercellular adhesion molecule-1 (ICAM-1) was found to be involved in the extravasation of neutrophils from the sinusoids to the hepatocytes (34). The inhibition of ICAM-1 was also discovered to be highly effective in preventing neutrophil-induced liver injury (34). The chemokines $\mathrm{C}-\mathrm{C}$ motif chemokine ligand 2, $\mathrm{C}-\mathrm{X}-\mathrm{C}$ chemokine ligand 2 , high mobility group protein $\mathrm{B} 1$, osteopontin, Il17, Il33, Ill $\beta$ and Tnf $\alpha$ have been identified to be involved in the chemotaxis of neutrophils (15,35-39). Certain proteins of the inflammatory pathway, such as ERK, p38, JNK, NF- $\mathrm{BB}$ and STAT3 are also reportedly involved in the production of chemokines $(9,11,40,41)$. However, to the best of our knowledge, the type of chemokine and the exact inflammatory pathways that are the most critical in the inflammatory process have not been fully identified. Moreover, the group of cells that produces these chemokines has not been completely discovered. Thus, these research questions remain to be answered.

In conclusion, the ANIT-induced cholestasis promoted a preferential disruption of the biliary system and triggered a stronger adaptation to BA metabolism, whereas the LCA-induced cholestasis mainly induced hepatocyte toxicity; therefore, the treatments were cholestatic and hepatocellular in nature, respectively, due to their different etiological mechanisms. However, the progression of chemotaxis and the development of inflammation exhibited certain similarities between these two models. The data also suggested that the JNK/STAT3 pathway was significantly activated in the two cholestatic models despite their different toxicity phenotypes, suggesting the same potential treatment target of the two different types of cholestatic liver injuries.

\section{Acknowledgements}

Not applicable.

\section{Funding}

The present study was supported by the Ningbo Natural Science Foundation (grant nos. 2018A610253 and 2018A610384), Zhejiang Public Welfare Technology Research Program (grant nos. LGD19H070001 and LY20H030001) and the K.C. WongMagna Fund in Ningbo University.

\section{Availability of data and materials}

The datasets used and/or analyzed during the current study are available from the corresponding author on reasonable request.

\section{Authors' contributions}

AL and JY designed the study and revised the manuscript; GX and MD performed the experiments, analyzed the data and wrote the manuscript; and XZ and HL contributed to the experimental work. All authors read and approved the final manuscript. 


\section{Ethics approval and consent to participate}

The animal studies were performed following the approval of the protocol by the Institutional Animal Care and Use Committee (approval no. IACUC 201707-138) at Ningbo University (Zhejiang, China).

\section{Patient consent for publication}

Not applicable.

\section{Competing interests}

The authors declare that they have no competing interests.

\section{References}

1. Hirschfield GM, Heathcote EJ and Gershwin ME: Pathogenesis of cholestatic liver disease and therapeutic approaches Gastroenterology 139: 1481-1496, 2010.

2. Poupon R: Primary biliary cirrhosis: A 2010 update. J Hepatol 52: 745-758, 2010.

3. Liu H, Liu Y, Wang L, Xu D, Lin B, Zhong R, Gong S, Podda M and Invernizzi P: Prevalence of primary biliary cirrhosis in adults referring hospital for annual health check-up in Southern China. BMC Gastroenterol 10: 100, 2010.

4. Lindkvist B, Benito de Valle M, Gullberg B and Bjornsson E: Incidence and prevalence of primary sclerosing cholangitis in a defined adult population in Sweden. Hepatology 52: 571-577, 2010.

5. Claessen MM, Vleggaar FP, Tytgat KM, Siersema PD and van Buuren HR: High lifetime risk of cancer in primary sclerosing cholangitis. J Hepatol 50: 158-164, 2009.

6. Tischendorf JJ, Hecker H, Kruger M, Manns MP and Meier PN: Characterization, outcome, and prognosis in 273 patients with primary sclerosing cholangitis: A single center study. Am J Gastroenterol 102: 107-114, 2007.

7. Zhang A, Jia Y, Xu Q, Wang C, Liu Q, Meng Q, Peng J, Sun H, Sun P, Huo X and Liu K: Dioscin protects against ANIT-induced cholestasis via regulating Oatps, Mrp2 and Bsep expression in rats. Toxicol Appl Pharmacol 305: 127-135, 2016.

8. Zhou HQ, Liu W, Wang J, Huang YQ, Li PY, Zhu Y, Wang JB, Ma X, Li RS, Wei SZ, et al: Paeoniflorin attenuates ANIT-induced cholestasis by inhibiting apoptosis in vivo via mitochondria-dependent pathway. Biomed Pharmacother 89 : 696-704, 2017.

9. Tan Z, Liu A, Luo M, Yin X, Song D, Dai M, Li P, Chu Z, Zou Z, Ma M, et al: Geniposide inhibits alpha-naphthylisothiocyanate-induced intrahepatic cholestasis: The downregulation of STAT3 and NF[formula: See text]B signaling plays an important role. Am J Chin Med 44: 721-736, 2016.

10. Connolly AK, Price SC, Connelly JC and Hinton RH: Early changes in bile duct lining cells and hepatocytes in rats treated with alpha-naphthylisothiocyanate. Toxicol Appl Pharmacol 93: 208-219, 1988 .

11. Dai M, Yang J, Xie M, Lin J, Luo M, Hua H, Xu G, Lin H, Song D, Cheng Y, et al: Inhibition of JNK signalling mediates PPARalpha-dependent protection against intrahepatic cholestasis by fenofibrate. Br J Pharmacol 174: 3000-3017, 2017.

12. Li X, Liu R, Yu L, Yuan Z, Sun R, Yang H, Zhang L and Jiang Z: Alpha-naphthylisothiocyanate impairs bile acid homeostasis through AMPK-FXR pathways in rat primary hepatocytes. Toxicology 370: 106-115, 2016.

13. Zeng H, Li D, Qin X, Chen P, Tan H, Zeng X, Li X, Fan X, Jiang Y, Zhou Y, et al: Hepatoprotective effects of schisandra sphenanthera extract against lithocholic acid-induced cholestasis in male mice are associated with activation of the pregnane $X$ receptor pathway and promotion of liver regeneration. Drug Metab Dispos 44: 337-342, 2016.

14. Zhang X, Ma Z, Liang Q, Tang X, Hu D, Liu C, Tan H, Xiao C, Zhang B, Wang Y, et al: Tanshinone IIA exerts protective effects in a LCA-induced cholestatic liver model associated with participation of pregnane X receptor. J Ethnopharmacol 164: 357-367, 2015.
15. Woolbright BL, Li F, Xie Y, Farhood A, Fickert P, Trauner M and Jaeschke H: Lithocholic acid feeding results in direct hepato-toxicity independent of neutrophil function in mice. Toxicol Lett 228: 56-66, 2014.

16. El-Agamy DS, Almaramhy HH, Ahmed N, Bojan B, Alrohily WD and Elkablawy MA: Anti-inflammatory effects of vardenafil against cholestatic liver damage in mice: A mechanistic study. Cell Physiol Biochem 47: 523-534, 2018.

17. Kong Y, Gao X, Wang C, Ning C, Liu K, Liu Z, Sun H, Ma X, Sun P and Meng Q: Protective effects of yangonin from an edible botanical Kava against lithocholic acid-induced cholestasis and hepatotoxicity. Eur J Pharmacol 824: 64-71, 2018.

18. Dai M, Hua H, Lin H, Xu G, Hu X, Li F, Gonzalez FJ, Liu A and Yang J: Targeted metabolomics reveals a protective role for basal PPAR $\alpha$ in cholestasis induced by $\alpha$-naphthylisothiocyanate. J Proteome Res 17: 1500-1508, 2018.

19. Livak KJ and Schmittgen TD: Analysis of relative gene expression data using real-time quantitative PCR and the 2(-Delta Delta C(T)) method. Methods 25: 402-408, 2001.

20. Melanie S, Benedikt K, Pfaffl MW and Irmgard R: The potential of circulating extracellular small RNAs (smexRNA) in veterinary diagnostics-Identifying biomarker signatures by multivariate data analysis. Biomol Detect Quantif 5: 15-22, 2015.

21. Waner T and Nyska A: The toxicological significance of decreased activities of blood alanine and aspartate aminotransferase. Vet Res Commun 15: 73-78, 1991.

22. Ning T, Guo J, Zhang K, Li K, Zhang J, Yang Z and Ge Z: Nanosecond pulsed electric fields enhanced chondrogenic potential of mesenchymal stem cells via JNK/CREB-STAT3 signaling pathway. Stem Cell Res Ther 10: 45, 2019.

23. Golbar HM, Izawa T, Yano R, Ichikawa C, Sawamoto O, Kuwamura M, Lamarre J and Yamate J: Immunohistochemical characterization of macrophages and myofibroblasts in $\alpha$-Naphthylisothiocyanate (ANIT)-induced bile duct injury and subsequent fibrogenesis in rats. Toxicol Pathol 39: 795-808, 2011.

24. Lleo A, Maroni L, Glaser S, Alpini G and Marzioni M: Role of cholangiocytes in primary biliary cirrhosis. Semin Liver Dis 34: 273-284, 2014

25. Deo AK and Bandiera SM: Biotransformation of lithocholic acid by rat hepatic microsomes: Metabolite analysis by liquid chromatography/mass spectrometry. Drug Metab Dispos 36: 442-451, 2008.

26. Aithal GP, Watkins PB, Andrade RJ, Larrey D, Molokhia M, Takikawa H, Hunt CM, Wilke RA, Avigan M, Kaplowitz N, et al: Case definition and phenotype standardization in drug-induced liver injury. Clin Pharmacol Ther 89: 806-815, 2011.

27. Meng Q, Chen XL, Wang CY, Liu Q, Sun HJ, Sun PY, Huo XK, Liu ZH, Yao JH and Liu KX: Alisol B 23-acetate protects against ANIT-induced hepatotoxity and cholestasis, due to FXR-mediated regulation of transporters and enzymes involved in bile acid homeostasis. Toxicol Appl Pharmacol 283: 178-186, 2015.

28. Miyata M, Watase H, Hori W, Shimada M, Nagata K, Gonzalez FJ and Yamazoe Y: Role for enhanced faecal excretion of bile acid in hydroxysteroid sulfotransferase-mediated protection against lithocholic acid-induced liver toxicity. Xenobiotica 36: 631-644, 2006.

29. Bodin K, Lindbom U and Diczfalusy U: Novel pathways of bile acid metabolism involving CYP3A4. Biochim Biophys Acta 1687: 84-93, 2005

30. Lu TT, Makishima M, Repa JJ, Schoonjans K, Kerr TA, Auwerx J and Mangelsdorf DJ: Molecular basis for feedback regulation of bile acid synthesis by nuclear receptors. Mol Cell 6: 507-515, 2000.

31. Sun L, Xie C, Wang G, Wu Y, Wu Q, Wang X, Liu J, Deng Y, Xia J, Chen B, et al: Gut microbiota and intestinal FXR mediate the clinical benefits of metformin. Nat Med 24: 1919-1929, 2018.

32. Yu SJ, Bae S, Kang JS, Yoon JH, Cho EJ, Lee JH, Kim YJ, Lee WJ, Kim CY and Lee HS: Hepatoprotective effect of vitamin $\mathrm{C}$ on lithocholic acid-induced cholestatic liver injury in Gulo(-/-) mice. Eur J Pharmacol 762: 247-255, 2015.

33. Roth RA and Dahm LJ: Neutrophil- and glutathione-mediated hepatotoxicity of alpha-naphthylisothiocyanate. Drug Metab Rev 29: 153-165, 1997.

34. Essani NA, Fisher MA, Farhood A, Manning AM, Smith CW and Jaeschke H: Cytokine-induced upregulation of hepatic intercellular adhesion molecule-1 messenger RNA expression and its role in the pathophysiology of murine endotoxin shock and acute liver failure. Hepatology 21: 1632-1639, 1995. 
35. Woolbright BL and Jaeschke H: Therapeutic targets for cholestatic liver injury. Exp Opin Ther Targets 20: 463-475, 2016.

36. Woolbright BL and Jaeschke H: Inflammation and cell death during cholestasis: The evolving role of bile acids. Gene Expr 19: 215-228, 2019.

37. Lou G, Ma X, Fu X, Meng Z, Zhang W, Wang YD, Van Ness C, Yu D, Xu R and Huang W: GPBAR1/TGR5 mediates bile acid-induced cytokine expression in murine Kupffer cells. PLoS One 9: e93567, 2014.

38. Cai SY, Ouyang X, Chen Y, Soroka CJ, Wang J, Mennone A, Wang Y, Mehal WZ, Jain D and Boyer JL: Bile acids initiate cholestatic liver injury by triggering a hepatocyte-specific inflammatory response. JCI Insight 2: e90780, 2017.
39. Woolbright BL, Dorko K, Antoine DJ, Clarke JI, Gholami P, Li F, Kumer SC, Schmitt TM, Forster J, Fan F, et al: Bile acid-induced necrosis in primary human hepatocytes and in patients with obstructive cholestasis. Toxicol Appl Pharmacol 283: 168-177, 2015.

40. Li C, Yan Y, Shi Q, Kong Y, Gao L, Bao H and Li Y: Recuperating lung decoction attenuates inflammation and oxidation in cigarette smoke-induced COPD in rats via activation of ERK and Nrf2 pathways. Cell Biochem Funct 35: 278-286, 2017.

41. Zhang W, Dai H, Lin F, Zhao C, Wang X, Zhang S, Ge W, Pei S and Pan L: Ly-6C(high) inflammatory-monocyte recruitment is regulated by $\mathrm{p} 38 \mathrm{MAPK} / \mathrm{MCP}-1$ activation and promotes ventilator-induced lung injury. Int Immunopharmacol 78: 106015, 2020. 